\title{
Surgical Approach To Manage Cerebrospinal Fluid Rhinorrhea Through Vault Or Through Nose
}

\author{
Nabeel Humayun Hassan, Ahmad Nawaz Ahmad, Zain. A. Sobani, Anwar Suhail, Rahila Usman
}

- - - - - - - - - - - - - - - - - - - - - - - - - - - - -

ABSTRACT

Objective: Background: Surgical management of cerebrospinal fluid (CSF) rhinorrhea can be done through a transcranial approach or endoscopically using a transnasal approach. The endoscopic technology is relatively fresh in developing countries. Keeping this in mind we conducted an audit of patients undergoing endoscopic repair of CSF leaks to review their outcome in terms of recurrence and complications and compare them with the patients had transcranial repair. The objective of the study is to review the management of patients who underwent repair of CSF rhinorrhea at Lyari General Hospital, Aga Khan University Hospital and Memon Medical Institute Hospital - 10 years experience.

Study design: Cross-sectional observational study

Place and duration of Study: Lyari General Hospital, Aga Khan University Hospital and Memon Medical Institute Hospital, from January 2005 to December 2014

Patients \& methods: A review of patient charts having undergone surgical repair for CSF rhinorrhea in the last 10 years at our institution was conducted. Thirty eight patients meeting the inclusion criteria of having undergone a surgical procedure for the repair of CSF rhinorrhea with a minimum post operative follow up of 6 months were included in the study.

Results: Skull base defects were repaired with the help of minimally invasive transnasal endoscopic approach with a success rate of $80 \%$ in comparison to transcranial repair success rate of $29 \%$. Post-operative complications were seen in only $10 \%$ of endoscopic group and $53 \%$ of transcranial group.

Conclusion: Although endoscopic management is associated with better outcomes there is room for improvement in the approach in developing countries and training programs and detailed internal audits need to be conducted to improve the situation to the level of developed countries.

Keywords: Endoscopic repair, cerebrospinal fluid rhinorrhea, minimally invasive, endoscope

\section{INTRODUCTION:}

Cerbrospinal fluid (CSF) rhinorrhea is a rare but potentially lethal condition. It was first described by Galen in 2000 B.C. ${ }^{1}$ It is currently defined as a condition involving the leakage of CSF from the subarachnoid space via a skull base defect into the paranasal sinuses and eventually the nose. ${ }^{2}$ The potential sites of the CSF leak include the cribriform plate (70-80\%) and roof of the ethmoid sinus (5$10 \%$ ). Omaya et. al, in the year 1968 classified the condition into two major groups (traumatic and non traumatic) based

Nabeel Humayun Hassan, $---\div-----1$

I Assistant Professor, ENT

I Shaheed Mohtarma Benazir Bhutto Medical College, Lyari

General Hospital

Email address: nabeelkmdcian@yahoo.com

I

Ahmad Nawaz Ahmad,

Assistant Professor,

I Liaquat National Medical College and Hospital

I Zain. A. Sobani,

I Residnet, Medical College Aga Khan University.

I Anwar Suhail,

I Assistant Professor, ENT Aga Khan University Hospital.

I Rahila Usman,

I Assistant Professor, Radiology Department,

I Dow University of Health Sciences

I Received: 06-07-18

Accepted: 13-08-18 on the etiology. The traumatic group was further subdivided into accidental and surgical trauma, whereas the non traumatic were further divided based on the presence or absence of elevated intracranial pressure. ${ }^{3}$

Various strategies in the management of this condition have been adopted over time. Thompson et. al, reported the first series of patients suffering from spontaneous CSF leaks managed conservatively in $1889 .{ }^{4}$ A variety of management methods has been described with variable outcomes. ${ }^{5}$ The first successful procedure to stop CSF rhinorrhea was carried out by Dandy Walker in the year 1926 when he approached the defect via a bi-frontal craniotomy and sutured fascia lata over the defect. ${ }^{6}$ However the approach was associated with a risk of serious complications including anosmia, intracranial hemorrhage, meningitis and death in rare cases.

Dandy's intracranial approach was followed by Dohlman's naso-orbital approach, which became the first extra cranial approach described in literature. ${ }^{7}$ Hirsch in 1952 became the pioneers of the transnasal route. ${ }^{8}$ Following their footsteps Wigand repaired a number of defects in the cribriform plates and sphenoidal sinus using rigid endoscopes in 1981, ${ }^{9}$ leading to the popularization of this minimally invasive approach.

Recurrence and complication rates have been quite variable in different regions and settings. A meta-analysis of 14 
studies showed success rate approaching $92 \%$. This figure has improved since then; Rodney et. al, reported a success rate close to $96 \%$. These high success rates along with minimal risks of complications have made the endoscopic transnasal procedure the primary choice for patients requiring surgical repair of uncomplicated CSF rhinorrhea. However there are a few shortcomings in this approach which limit its application. The situations where in it may not be successful include high pressure leaks, multiple skull base defects and cases associated with intracranial pathologies.

The technique is fairly new in developing countries and data regarding its application in the developing world is scarce. In this regard, we conducted a retrospective review of patients undergoing endoscopic repair of CSF rhinorrhea with the objective to review their outcome in terms of recurrence and complications and compare them with patients who underwent transcarnial repair.

\section{METHODS:}

This was a cross sectional observational study. We retrospectively reviewed charts of patients undergoing surgical repair of a CSF leak either transcranilly or endoscopically at different centers mentioned above from the year Jan 2005 to December 2014. All the patients underwent surgical repair of CSF leak during the above mentioned period were included however the patients with associated meningioencephalocele or any intracranial space occupying lesion or had any prior surgical treatment for this condition were excluded. The patients having follow up of less than 6 months or had incomplete records were also excluded. Fifty three patients were identified in our health information management system, of which 15 cases were excluded.

All case notes, records, investigations, method of repair and outcome in terms of failure of repair and complications of the included 38 patients were reviewed and the data was recorded in a predesigned database. Complications were recorded along with the predisposing factors and measures taken for their prevention and management.

The data was analyzed using Statistical Package for Social Sciences version 19 (SPSSv19.0). Mean and standard deviation for continuous variables was computed. Frequency $\&$ percentages were computed for categorical variables. The statistical analysis was done using chi-square test taking pvalue of $<0.05$ as significant with confidence interval of 95\%.

\section{RESULTS:}

The study population was predominantly female with a male to female ratio of 1:1.7, with a median age of $40 \pm 20$ years (Range 3-61 years).

The most common presenting complaint was watery nasal discharge $65 \%$ of which $60 \%$ was unilateral and $40 \%$ was bilateral. Recurrent meningitis was the presenting complaint in $23.6 \%$. Iatrogenic and non iatrogenic trauma accounted for $15.8 \%$ and $57.9 \%$ of cases respectively. Spontaneous CSF rhinorrhea was present in $26.3 \%$ of cases. Few patients also have associated anosmia.

The site of the leak was determined using computed tomography (CT) with or without intrathecal contrast or magnetic resonance imaging (MRI). The procedure for diagnosis was not standardized as our center is a major referral center in the city and a significant proportion of the patients were diagnosed elsewhere and referred to our center for surgical management. In the cases diagnosed using CT scans the site of leak was identified in $50 \%$ of cases, the MRI showed superior results in this regard identifying the leak in $86.59 \%$ of patients.

About half, $52.6 \%$ of the cases had a defect in the cribriform plate, while the others showed defects in the sphenoid bone, fovea ethmoidalis and frontal bone (table 1). Nearly $63.1 \%$ of patients suffered from small sized $(<5 \mathrm{~mm})$ leaks, while $23.6 \%$ and $13.1 \%$ suffered from medium and large leaks respectively (table 1). The patients were evenly distributed in both groups with regards to the size of the leak. Three of our patients also had an associated meningiocele, one of which was managed using a minimally invasive endoscopic approach. Both of these two groups were stratified according to cause of CSF Rhinorrhea, size and site of leak, the recurrence for each factor was also analysed using chisquare and none of them was statistically significant to effect recurrence rate as shown in table I.

The most common material used for repairing the defect was autologous fascia used in $71 \%$ of cases, other materials included fat $26.3 \%$ and turbinate $2 \%$.

Among thirty eight patients minimally invasive endoscopic method of CSF repair was used in 10 patients and rest of 28 patients had transcranial repair, with failure rate of $10 \%$ and $29 \%$ respectively.

The average duration of hospital stay was only 4 days for patients had repair through minimally invasive approach but it was 7 days on average for patients required craniotomy. The complication rates was also reviewed and only two patients in endoscopic had complications that constituted $10 \%$ of study population in comparison to $53 \%$ in transcranial group.

\section{DISCUSSION:}

A large number of audits and reviews have been published on CSF rhinorrhea to date, most of which include limited sample sizes due to the rarity of the condition. ${ }^{10,11,12}$ Kirtane et. al, in their review of 267 patients reported that the two most common presenting complaints were rhinorrhea and recurrent meningitis. ${ }^{13}$ Most of their cases were attributable to trauma with the cribriform plate being the commonest site of involvement. ${ }^{14}$ Other than cribriform plate fovea ethmoidalis, roof of sphenoid sinus and frontal sinus wall 
Nabeel Humayun Hassan, Ahmad Nawaz Ahmad, Zain. A. Sobani, Anwar Suhail, Rahila Usman

\begin{tabular}{|c|c|c|c|c|}
\hline \multicolumn{5}{|l|}{ Site of Defect } \\
\hline & Sphenoid & Cribriform plate & Fovea ethmoidalis & Frontal \\
\hline Endoscopic approach & 6 & 2 & \begin{tabular}{|c|}
2 \\
\end{tabular} & 0 \\
\hline Intracranial approach & 5 & 18 & 3 & 2 \\
\hline Total & 11 & 20 & 5 & 2 \\
\hline Recurrence & 3 & 4 & 1 & 2 \\
\hline p-value & \multicolumn{4}{|c|}{0.106} \\
\hline \multicolumn{5}{|c|}{ Causes of Cerebrospinal Fluid Rhinorrhea } \\
\hline & Trauma & Spontaneous & Iatrogenic & \\
\hline Endoscopic approach & 4 & 3 & 3 & \\
\hline Intracranial approach & 18 & 7 & 3 & \\
\hline Total & 22 & 10 & 6 & \\
\hline Recurrence & 8 & 1 & 1 & \\
\hline p-value & & 0.453 & & \\
\hline \multicolumn{5}{|l|}{ Size of defect } \\
\hline & Small & Medium & Large & \\
\hline Endoscopic approach & 5 & 5 & 1 & \\
\hline Intracranial approach & 19 & 4 & 4 & \\
\hline Total & 24 & 9 & 5 & \\
\hline Recurrence & 7 & 1 & 2 & \\
\hline \multicolumn{4}{|l|}{ p-value } & \\
\hline
\end{tabular}

Table I: Repair methods used for different types of leaks and their outcome

leak can be the contributing factor ${ }^{11,14}$ A similar trend was seen in our setting with regard to presenting complaints, site of involvement and causes as reported in literature. ${ }^{9-12}$ Although trauma was the underlying cause for nearly 3 quarters of our patient population, only $16 \%$ of the cases were due to iatrogenic trauma. Of the $16 \%$ half had undergone a transnasal hypophysectomy; which has been described as the commonest cause of iatrogenic trauma leading to CSF rhinorrhea, ${ }^{15}$ and is regarded as an inherent manifestation of the operating protocol. The cribriform plate was commonest site of the defect in our series of patients accounting for $52 \%$ of leaks, this could be attributable to the fact that the cribriform plate is by nature the thinnest and most vulnerable bone in the anterior skull base and that it is already perforated by the olfactory nerve roots.

A major portion of our population suffered from small sized leaks; however a significant number also had medium and large sized leaks and they were also dealt smoothly. We found no relation of size with the outcome of the procedure and were able to successfully repair leaks endoscopically regardless of size of the defect.

A number of surgical and non-surgical methods were described in literature for the management of CSF Rhinorrhea. Recurrence vs complications were the major outcome compared in most of the literature. ${ }^{16,17,18}$

Among the two surgical techniques the endoscopic trans nasal approach was a less inavasive described technique with learning curve the outcomes can be achieved comparable to that of the orthodox craniotomy approach. ${ }^{19,20,21,22} \mathrm{We}$ found a significant low complication rate and less post operative stay in our patients. The cases which underwent endoscopic repair also had a lesser chance of relapse on follow up with a success rate of $80 \%$. Of interest to note here is that although the success rate for intra cranial repair has been internationally reported up to $90 \%$. $^{20,21,22}$ A series from our center had shown the success rate of endoscopic repair approaching up to $70 \%$ which included the patients till 2011. ${ }^{23}$ Although we have improved our success rate but it is still lower when compared to those reports by the developed world which are nearly $98 \%{ }^{24}$ The reason behind this deficit may be the relative lack of experience with the technique as it is fairly recent in the developing world, but growing experience has improved the outcome, as in some centers endoscopic CSF Rhinorrhea repair is also done on day care basis showing high level of expertise. In our study the high success rate may however be an exaggerated figure due to our limited sample size.

\section{CONCLUSION:}

The authors would like to conclude by stating that although a transnasal endoscopic approach to repair CSF rhinorrhea is a feasible management strategy with a better outcome than intracranial procedures to achieve the same, there is room for improvement in the approach in developing countries and comprehensive training programs and detailed internal audits need to be conducted to improve the success rates and make them comparable to those quoted by centers 
in the developed world.

\section{REFERENCES:}

1. Araujo Filho BC, Butugan O, Pádua FG, et al. Endoscopic repair of CSF rhinorrhea: experience of 44 cases. Braz J Otorhinolaryngol 2005; 71:472-6.

2. Chapter 55 Cerebrospinal Fluid Rhinorrhea, in Cumming's Otolaryngology - Head \& Neck Surgery, Flint PW, et al. Editors 2010, Elsevier.

3. OmmayaAK. Cerebrospinal Fluid Rhinorrhea. Neurology 1964; 14:106-113.

4. ThomsonS. The Cerebrospinal Fluid: Its spontaneous escape from the nose., in Diseases of the nose and throat 1899, Cassell \& Co: London. p. 845.

5. Mathias T, Levy J, Fatakia A, McCoul ED. Contemporary Approach to the Diagnosis and Management of Cerebrospinal Fluid Rhinorrhea. Ochsner J. 2016;16:136-42.

6. DandyW. Pneumocephalus (Inracranial pneumato- cele or aerocele). Arch Surg 1926; 12:949-982.

7. Dohlman G. Spontaneous cerebrospinal rhinorrhea. Acta Otolaryngol Suppl 1948; 67:20-23.

8. HirschO. Successful closure of cerebrospinal fluid rhinorrhea by endonasal surgery. Arch Otolaryngol 1952; 56:1-13.

9. Wang L, J Kim, CB Heilman. Intracranial mucocele as a complication of endoscopic repair of cerebrospinal fluid rhinorrhea: case report. Neurosurgery 1999; 45:1243-1245.

10. Tumturk A, Ulutabanca H, Gokoglu A, Oral S, Menku A, Kurtsoy A. The Results of the Surgical Treatment of Spontaneous Rhinorrhea via Craniotomy and the Contribution of CT Cisternography to the Detection of Exact Leakage Side of CSF. Turk Neurosurg. 2016;26(5):699-703.

11. Albu S, Florian IS, Bolboaca SD. The benefit of early lumbar drain insertion in reducing the length of CSF leak in traumatic rhinorrhea. Clin Neurol Neurosurg. 2016 Mar;142:43-47.

12. Amer A. Al-Shurbaji, Zuhair A. Abu Salma, Rami Y., A1Qroom, Anas Said. Spontaneous CSF Rhinorrhea: Management Protocol according to clinical presentation. JRMS Aug 2017; 24(2):70-74

13. Ulrich MT, Loo LK, Ing MB. Recurrent CSF Rhinorrhea Misdiagnosed as Chronic Allergic Rhinitis with Subsequent Development of Bacterial Meningitis. Case Rep Med. 2017;2017:9012579

14. Kirtane, MV, K. Gautham, SR Upadhyaya. Endoscopic CSF rhinorrhea closure: our experience in 267 cases. Otolaryngol Head Neck Surg 2005; 132:208-212.
15. Mirza S, Thaper A, McClelland L, et al. Sinonasal cerebrospinal fluid leaks: management of 97 patients over 10 years. Laryngoscope 2005; 115:1774-1777.

16. Scholsem M, Scholtes F, Collignon F, et al. Surgical management of anterior cranial base fractures with cerebrospinal fluid fistulae: a single-institution experience. Neurosurgery 2008; 62:463-469.

17. Jiang ZY, McLean C, Perez C, Barnett S, Friedman D, Tajudeen BA, Batra PS. Surgical Outcomes and Postoperative Management in Spontaneous Cerebrospinal Fluid Rhinorrhea. J Neurol Surg B Skull Base. 2018;79: 193-199.

18. Adams AS, Francis DO, Russell PT. Outcomes of outpatient endoscopic repair of cerebrospinal fluid rhinorrhea. Int Forum Allergy Rhinol. 2016;6(11):1126-1130

19. Sevinç Eraslan, Sercan Göde , Umut Erdoðan, Ýsa Kaya, Rapit Midilli , H. Bülent Karc. Endoscopic Cerebral Spinal Fluid (CSF) Rhinorrhea: Clinical Experiences. Eur J Rhinol Allergy 2018; 1(1): 1-4

20. Freyschlag CF, Goerke SA, Obernauer J, Kerschbaumer J, Thomé C1, Seiz M.A sandwich technique for prevention of cerebrospinal fluid rhinorrhea and reconstruction of the sellar floor after microsurgical transsphenoidal pituitary surgery. J Neurol Surg A Cent Eur Neurosurg. 2016;77(3):229-32

20. DeConde AS, Suh JD, Ramakrishnan VR. Treatment of cerebrospinal fluid rhinorrhea. Curr Opin Otolaryngol Head Neck Surg. 2015 Feb;23(1):59-64

21. Ibrahim AA, Okasha M, Elwany S. Endoscopic endonasal multilayer repair of traumatic CSF rhinorrhea. Eur Arch Otorhinolaryngol. 2016;273(4):921-6

22. Brandon Lucke-Wold, Erik C. Brown, Justin Cetas, Sachin Gupta, Timothy Hullar, Timothy Smith, Jeremy N. Ciporen. Minimally Invasive Endoscopic Repair of Refractory Lateral Skull Base Cerebral Spinal Fluid Leaks: A Summary and Technical Synthesis. J Neurol Surg B 2018; 79(S 01): S1S188

23. Muhammad Zubair Tahir, Muhammad Babar Khan, Muhammad Umair Bashir, Shabbir Akhtar,1 and Ehsan Bari. Cerebrospinal fluid rhinorrhea: An institutional perspective from Pakistan. Surg Neurol Int. 2011; 2:174

24. Banks CA, Palmer JN, Chiu AG, et al. Endoscopic closure of CSF rhinorrhea: 193 cases over 21 years. Otolaryngol Head Neck Surg 2009; 140:826-833.

25. Adams AS, Francis DO, Russell PT. Outcomes of outpatient endoscopic repair of cerebrospinal fluid rhinorrhea. Int Forum Allergy Rhinol. 2016;6(11):1126-1130 